\title{
INVESTIGACIONES
}

\section{ESTIMACION DE TIEMPO EN ESTUDIANTES SECUNDARIOS \\ CHILENOS FRENTE A UNA TAREA ESPACIAL DESARROLLADA EN FORMATOS DE PAPEL Y ORDENADOR}

\author{
Valuation of time in front of specifics tasks developed on paper and through personal \\ computers in high school's Chilean students
}

\author{
Luis Cárcamo Ulloa ${ }^{1}$, Ramón Cladellas Pros ${ }^{2}$, Santiago Estaún Ferrer ${ }^{3}$ \\ ${ }^{1}$ Universidad Austral de Chile, Instituto de Comunicación Social. lcarcamo@uach.cl \\ ${ }^{2}$ Universidad Autónoma de Barcelona, Departament de Psicologia Bàsica, \\ Evolutiva i de 1'Educació. Ramon.cladellas@uab.es \\ ${ }^{3}$ Universidad Autónoma de Barcelona, Departament de Psicologia Bàsica, \\ Evolutiva i de l'Educació. Santiago.estaun@uab.es
}

\section{Resumen}

El presente artículo da cuenta de los principales resultados de investigaciones experimentales desarrolladas en Chile con el objetivo de comparar la estimación del tiempo que hacen adolescentes cuando se enfrentan al desarrollo de tareas similares con o sin ordenadores. Además se analizan posibles diferencias culturales con grupos de sujetos rurales y urbanos.

La experiencia tuvo lugar entre los meses de abril y mayo de 2005 y los grupos de participantes provienen de colegios de la provincia de Valdivia en la zona sur de Chile.

Palabras clave: estimación de tiempo, cambio tecnológico, ordenadores en la escuela, Chile, percepción de tiempo, tecnología y cultura.

\begin{abstract}
The present article gives account of the main results of developed experimental investigations in Chile that it has by objective to compare the estimation of the time which adolescents do when they face the development of similar tasks with without computers. In addition possible cultural differences with groups of rural and urban subjects are analyzed.

The experience took place between the months of April and May of the 2005 and the groups of participants come from schools of the province of Valdivia in the south of Chile.

Key words: estimation of time, technological change, computers in the school, Chile, perception of time, technology and culture.
\end{abstract}




\section{PLANTEAMIENTO TEORICO}

La relación Hombre-Tecnología. La relación hombre-tecnología es revisada por algunos autores como un proceso de creación de artefactos para el dominio de la naturaleza que luego se transforman en extensiones de la mente humana (Mc Luhan 1990). En un sentido más ligado a una mirada economicista, Schwartz (2001) plantea el concepto de darwinismo digital para explicar cómo el hombre requiere de habilidades adaptativas para sobrevivir en la nueva economía digital. Desde una perspectiva más sociocultural, Castells $(1997$; 2001) plantea que el cambio hacia una sociedad red implica aspectos laborales, comunicacionales y de creación de nuevas identidades provocadas por las interacciones mediadas por la red de redes.

Toledo y Comba (2002) reconocen -a partir de sus investigaciones con profesionales que han vivido el impacto del cambio tecnológico en los años noventa- dos epistemologías para comprender la relación del hombre con las Tecnologías de la Información y las Comunicaciones. Y, en un esfuerzo por explicar cómo el hombre y la mujer se relacionan con las máquinas que crean, Toledo y Comba (2002) explican situaciones mediadas por ordenadores en las que el hombre es capaz de rehacerse a sí mismo, dan forma a nuevas socialidades y modos de conocer; es decir, "modos de hacer y de ser en el mundo".

- Una episteme naturalista, que caracteriza a los profesionales que comparten una formación académica, un ambiente y unas prácticas relacionadas con las ciencias exactas. Para estos profesionales, la computadora no produjo un cambio substancial en su trabajo, interactúan con la máquina dándole órdenes, ya que ésta sin la intervención humana no les aportó demasiado.

- Una episteme humanista, que caracteriza a los profesionales, que comparten una formación académica, un ambiente y unas prácticas relacionadas con las ciencias humanas. Para estos profesionales, la computadora se convirtió en una herramienta esencial para su trabajo, al punto tal que les resulta difícil establecer una separación entre ellos y la máquina. La interacción con la computadora adoptó la forma de una conversación en el transcurso de la cual el proceso creativo va transcurriendo mediante lo cooperación (Toledo y Comba 2002: 23).

Toledo y Comba (2002) concluyen, en buena medida, que el trato anterior al impacto tecnológico que tenían ciertos ejes profesionales determina en buena parte la nueva relación en la actual era de la información. Mientras para los ingenieros, por ejemplo, la tecnología no es una sorpresa y sigue siendo una máquina de cálculo, para los humanistas pasa a ser un mundo nuevo a descubrir y un "sombrero de mago" maravilloso.

En este contexto, resulta interesante plantearse si estas percepciones encontradas en grupos de profesionales son similares o diferentes en grupos de adolescentes que tienen en la actualidad un acercamiento tecnológico más temprano e integrado a sus mundos sociales.

Inserción de las tecnologías en la escuela. En Chile, el proyecto llamado Red Enlaces es la estrella de los gobiernos de la Concertación, coalición integrada por democratacristianos y socialdemócratas con 15 años en el poder postdictadura. La iniciativa -desde 
su inicio- en el año 1991 hasta el año 2005 ha logrado alcanzar importantes índices de cobertura de acceso a computadores. Así, para la Educación Primaria (Educación General Básica) el proyecto Enlaces alcanza a un $94 \%$ de los niños y niñas escolarizadas. En el caso de la Educación Secundaria (Educación Media), la cobertura se da en un $87 \%$ de los liceos. Ante esta realidad, los investigadores en educación coinciden en recalcar la importancia y necesidad de incorporar las tecnologías de forma adecuada en escuelas y liceos.

Se hace un llamado de atención a la escuela para que tome las medidas correspondientes para preparar a todos los estudiantes en el manejo de los medios tecnológicos y virtuales, considerándose este aspecto como una característica de los tiempos que es necesario abordar en forma oportuna para evitar la marginación (Beas 2003: 49).

La brecha digital es una preocupación actual de todos los países en vías de desarrollo o que aspiran a un régimen general de vida mejor para sus ciudadanos. Es justamente la reducción de la brecha digital una de las principales metas del proyecto Enlaces. En ese sentido, las dificultades que ofrece la ruralidad de algunas regiones en Chile significa un desafío importante donde los recursos tecnológicos tratan de repartirse con equidad. Allí el $83 \%$ de las escuelas rurales tienen cobertura de la red enlaces, mientras que las escuelas urbanas alcanzan a un $91 \%$. "La política pública de generación de acceso a TIC a través de la integración de tecnología a escuelas, es el mecanismo que ha permitido equiparar las condiciones desiguales en el acceso que tienen las familias chilenas" (Enlaces 2005: 25).

Para los educadores, las preguntas se dirigen a ¿qué se debe enseñar con apoyo de la tecnología? Pero los aspectos que aparecen no se refieren necesariamente al desarrollo de competencias técnicas, sino más bien a perfiles humanísticos, fundamentales para la convivencia y la participación social, además del desarrollo de un rol más activo como estudiante.

Sin perder de vista esta dificultad y por cierto sin tener la pretensión de dar una respuesta exhaustiva contribuiremos a la discusión del tema centrándonos en tres aspectos que nos parecen relevantes: la educación para la convivencia democrática, la educación para la autonomía y la incorporación de la tecnología en los hábitos de los ciudadanos (Beas 2003: 52).

Con ello, se refuerza la hipótesis de Toledo y Comba (2002) que afirma que las tecnologías para el humanista resultan ser un valioso cajón de herramientas para la formación de dimensiones humanas muy diversas. Para autores como Josefina Beas (2003), las influencias de las tecnologías están más allá del currículo y se instalan prácticamente como el desarrollo de una actitud vital de autoformación permanente.

Percepción del Tiempo y Cambio Cultural. El cambio, según Hall (1989), podría entenderse como un fenómeno ante el cual el sujeto presenta una migración en sus actitudes formales, informales y técnicas. El ciclo lógico es que se pasa de un estado formal a uno informal; luego, como resultado dialéctico, se asume una actitud técnica analítica de la situación que está cambiando para asumir la nueva situación como formal. En la siguiente figura se puede esquematizar el proceso descrito. 


\section{Figura 1}

Transformación cíclica del tiempo. Fuente Propia

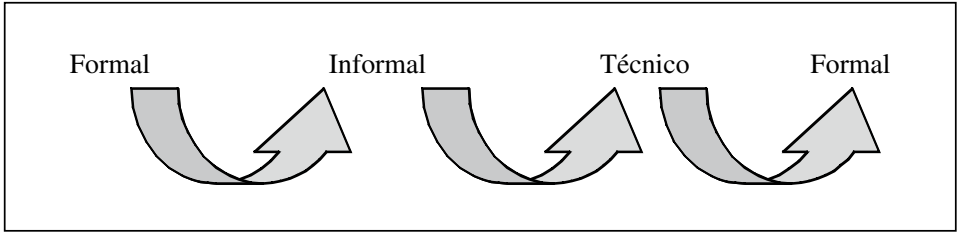

Las distintas culturas son análogas a las diferentes especies, en el sentido de que unas sobreviven en tanto que otras perecen. Algunas son más adaptables que otras. El estudio del cambio es, por lo tanto, el estudio de la supervivencia, así pues tiene algo más que un mero interés académico ver cómo lo formal, lo informal y lo técnico existen en una relación de cambio continuo. La teoría de la naturaleza de estas relaciones constituye una teoría del cambio (Hall 1989: 102).

Para dejar en claro el valor de los diversos modos de percibir el tiempo cabe señalar que, en otra línea específica de investigación como es el tiempo de reacción, Choudhury y Gorman (1999) cuestionan la validez predictiva cuando se asocia tiempo de reacción y mediciones de inteligencia. Los investigadores aclaran en sus conclusiones que se trataría más bien de que en las culturas rurales perciben el tiempo basándose en relaciones distintas de las que utilizan sujetos urbanos de grandes zonas industrializadas. Parece un factor repetido el hecho de que las culturas rurales perciban el tiempo con una mayor calma o lentitud, pero en ningún caso se debe ligar este aspecto a capacidades intelectuales.

$\mathrm{Si}$ el tiempo es un factor cultural de cambio, el hecho de que los distintos sujetos perciban mayor o menor presión o mayor o menor motivación frente a un cambio anunciado es también una forma de estudiar las diferencias culturales; un sujeto que sentirá o siente una presión de parte de la tecnología y que también demandará de ella respuestas eficientes. Todo esto también es parte de los tiempos que corren, los tiempos de la sociedad red. Motivación o presión constituyen las dos caras de un mismo cambio.

Convertido en dueño del tiempo, es decir, sabiendo medirlo y dosificarlo con gran precisión, economizarlo y gastarlo, el hombre también se encuentra dominado por él. En efecto, la fugacidad o irreversibilidad está continuamente presente en el "apresurado" hombre de nuestros días. La civilización contemporánea ha visto crecer inconmensurablemente el valor y la importancia de la rapidez, así como la transformación radical del ritmo de vida. Este ritmo es para los habitantes de los países industrializados algo habitual e inevitable. El tiempo irreversible, vectorial y dividido en segmentos de igual tamaño; este tiempo de nuestros cronómetros, relojes, calendarios; el tiempo entendido como una forma de existencia de la materia, como duración pura, forma parte integrante de la imagen científica del tiempo, tal como se ha constituido en los últimos siglos y cuyas modificaciones actuales siguen dentro del marco del pensamiento científico (Gurevitch 1979: 261).

En la actualidad una de las finalidades más recurrentes para justificar procesos de cambios del mundo analógico al digital se orientan hacia un mejor dominio del tiempo. 
Tareas que se desarrollaban en papel y lápiz buscan su rápida transformación en formatos de ordenador sin medir muchas veces los efectos de esos cambios en los seres humanos. Más allá de una posición unívoca respecto de lo favorable o perjudicial que resultan estos procesos transformadores de la vida cotidiana, es necesario investigar cuáles son esos efectos reales.

Percepción del tiempo frente a la ejecución de tareas. Los trabajos clásicos en percepción del tiempo Piaget y Meylan-Backs (1971), Orsini (1971), Henry (1971), Fraisse (1971) y Piaget (1971) explican que los sujetos interactuando en distintas tareas estiman el transcurso del tiempo de forma diferente. Fraisse y Piaget valoran en forma distinta la velocidad de ejecución de la tarea. Mientras que para Jean Piaget la percepción de la velocidad es fundamental para la percepción del tiempo, para Paul Fraisse no lo es necesariamente y plantea que la percepción del tiempo debe entenderse como una síntesis compleja de factores, donde la velocidad es uno entre otros. En este sentido Francine Orsini (1971) parece inclinarse más hacia la línea de Fraisse.

Podemos señalar que la hipótesis de Piaget parece convenir un cierto tipo de ejecución. Fue probada sobre todo en situaciones que implican el desplazamiento de móviles y en los cuales el niño es espectador observador. Cuando el papel del niño se vuelve más activo, cabe preguntarse si la evolución genética será idéntica respecto de situaciones diferentes en las que el tiempo por medir pertenece al desarrollo mismo del comportamiento (Orsini 1971: 162).

En 1969 Robert Orstein (1969) introduce el factor memoria como elemento decisivo para la percepción del tiempo. Donde el tiempo percibido depende de la cantidad de espacio memoria requerida para la actividad. Una tarea más compleja y/o con mayor cantidad de estímulos requiere de un mayor espacio de memoria. Como consecuencia de esto el tiempo percibido parece prolongarse. El planteamiento de Orstein (1969) es conocido como storage-size model.

Figura 2

Número de estímulos ante un mismo periodo de tiempo. Fuente Propia

SITUACIONA

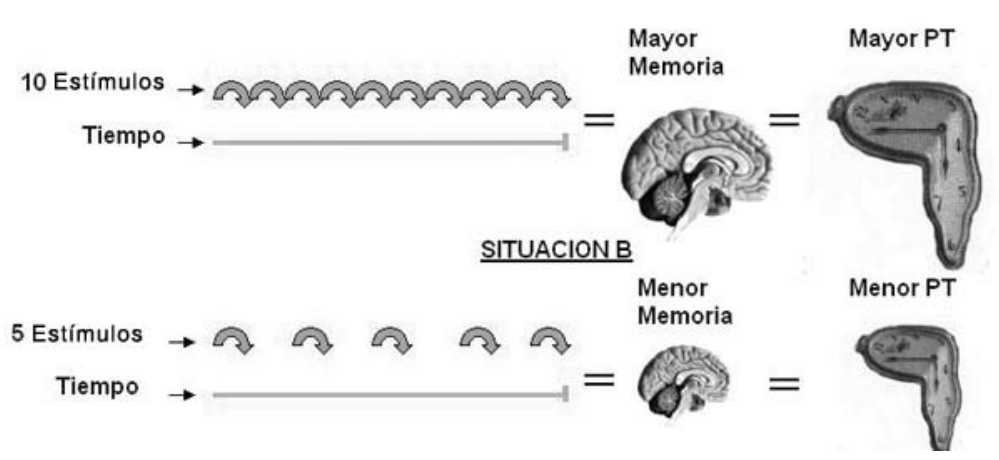


La figura 2 explica cómo el modelo de Orstein (1969) apunta a evaluar el rol de la memoria en la percepción del tiempo vivido, pero cabe tener la precaución de evaluar correctamente el peso de los estímulos, que no sólo se determina por la cantidad de los mismos, sino también por la calidad de ellos. La fórmula puede resultar muy consistente cuando los estímulos son de la misma naturaleza y calidad, pues demandarían una actividad cognitiva similar. Así como también es importante contemplar el problema de la acción del propio sujeto en la estimación del tiempo.

En 1989 Douglas Pointer propuso un modelo en el que el factor decisivo para la percepción del tiempo es el número de cambios, donde el cambio es el índice psicológico del paso del tiempo. Así cuando el intervalo se divide en un mayor número de segmentaciones se asumen como nuevos cambios y esto produce un efecto de elongación del tiempo. El planteamiento de Pointer (1989) es conocido como changesegmentation.

\section{Figura 3}

El tiempo segmentado por los cambios. Fuente Propia

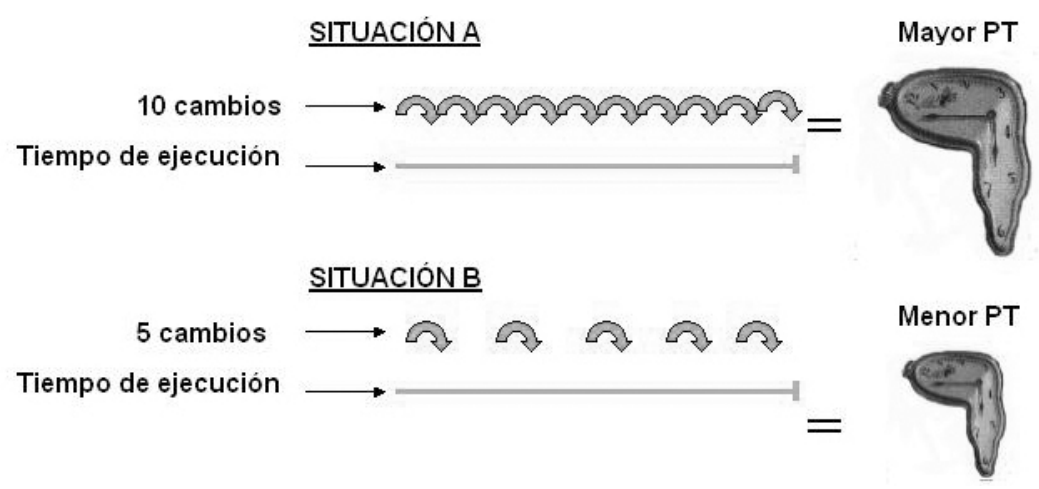

En la figura 3 los cambios segmentan el período del tiempo y esa segmentación produce que un mismo tiempo, en apariencia, se prolongue si el número de cambios es ostensiblemente mayor. Con ello resulta sumamente importante tener en cuenta que esta fórmula se repetirá mientras los cambios sean homogéneos.

Por su parte, Morales (2005) propone un modelo de tiempo psicológico en el estudiante, pensando en explicar los dos extremos del paso del tiempo en una sesión de estudio: La duración prolongada del tiempo y la compresión temporal.

Si concebimos cualquier posible percepción del tiempo mientras se estudia, el estudiante deberá experimentar algo entre un tiempo extremadamente lento (duración prolongada), a la sensación del tiempo que "pasa volando" (compresión temporal). Estas experiencias se dan en un marco de juicio tridimensional relacionadas a una autopercepción de la situación y las habilidades propias, así como a la carga que representa la tarea a realizar (Morales 2005: 21). 


\section{Figura 4}

Modelo para el tiempo psicológico en el estudiante (Morales 2005)

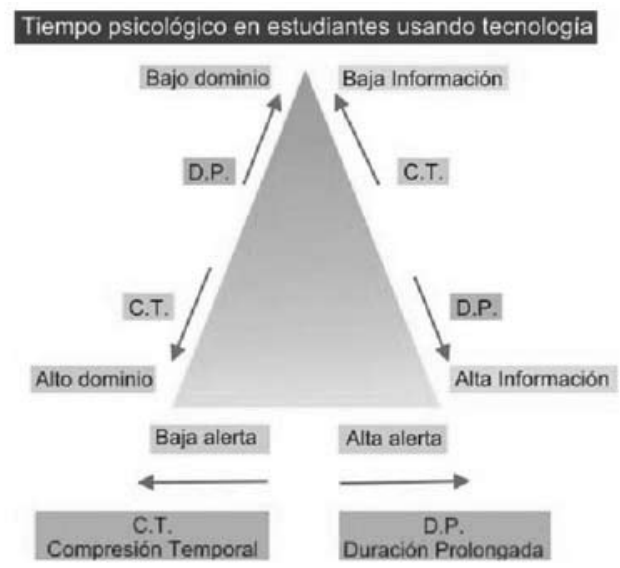

El modelo de Morales (figura 4) considera tres dimensiones (alerta, carga de información y dominio de la tarea) y las direcciones extremas del paso del tiempo (compresión temporal y duración prolongada). De esta forma la interacción entre las tres dimensiones determina el sentido de dirección del paso del tiempo comprimido o prolongado. Así, por ejemplo, si una tarea presenta alta alerta, alta carga de información y bajo dominio de la tarea tenderá a la percepción de una duración prolongada del tiempo.

Morales (2005) reconoce que el algoritmo descrito por su modelo es una derivación lógica simple de la combinación "alto-bajo" de variables y que las combinaciones extremas (alta-alta-alta, baja-baja-baja) se tratarían de estados inestables.

La lógica de situaciones reales implicaría combinatorias de alerta, dominio y carga de información y el modelo propuesto espera ser contrastado con actividades reales y el desarrollo de mediciones apropiadas que permitan predecir la percepción temporal de los estudiantes cuando están involucrados en tareas académicas. A continuación se presenta una situación experimental desarrollada con estudiantes chilenos de entre 15 y 17 años.

Las hipótesis y los objetivos que motivaron la presente investigación, desarrollada en su fase experimental con estudiantes de procedencias rurales y urbanas, son los que siguen:

\section{HIPOTESIS Y OBJETIVOS}

1. El formato de ejecución (ordenador o en papel) para una tarea espacial de resolución de diagramas incide en la estimación de tiempo que hacen los sujetos. Siendo sobreestimado el tiempo cuando la tarea se desarrolla en ordenador.

1a. El formato de ejecución (ordenador o en papel) para una tarea espacial de resolución de diagramas incide de forma distinta en la estimación de tiempo que hacen los 
sujetos que provienen de contextos culturales diferentes (rurales y urbanos). Siendo los sujetos rurales quienes estimen como menor el tiempo cuando desarrollan la tarea en ordenador.

El objetivo de este estudio es observar si se producen diferencias en la estimación de tiempo cuando una misma tarea espacial es ejecutada en ordenador o en papel. Con ello se buscan respuestas específicas a la introducción de tecnologías en contextos rurales y urbanos, contemplando las conclusiones de estudios anteriores sobre uso de ordenador y/o diferencias culturales en el caso de sujetos urbanos y rurales (Hall 1989; Choudhury y Gorman 1999).

A modo de medir diferencias de impacto de la introducción de tecnologías, se comparan grupos equivalentes de sujetos mayormente impactados (urbanos) y otros menor impactados (rurales). Se asumen grupos que, al comparar adolescentes del sistema educacional regular chileno, el impacto 0 de las tecnologías es imposible de hallar como sujetos de estudio. Esto basándose en las cifras de introducción y cobertura de tecnología del programa RED ENLACES del Ministerio de Educación de Chile. Situación que, como se ha detallado, alcanza un $87 \%$ para los liceos de educación secundaria. En el caso de las escuelas primarias rurales un $83 \%$ con laboratorios instalados y en las escuelas urbanas la cobertura alcanza al $93 \%$ de los establecimientos.

En términos de análisis estadístico del trabajo, se requiere comparar las diferencias de estimación de tiempo de los distintos conjuntos de sujetos (rurales-urbanos), siendo el tiempo estimado en las ejecuciones de ordenador y papel la variable dependiente.

\section{METODO}

Sujetos. La experiencia involucró a 58 sujetos, de los cuales 29 son de procedencia urbana y 29 rurales.

Tabla 1

Distribución de la población experimental por procedencia

\begin{tabular}{|c|c|c|c|}
\hline & Urbanos & Rurales & Total \\
\hline Sujetos & 29 & 29 & 58 \\
\hline
\end{tabular}

El trabajo experimental se desarrolló durante los meses abril y mayo de 2005 en el Liceo Rural People Help People de Pullinque en la provincia de Valdivia y los Liceos urbanos Salesianos y Liceo Técnico de Niñas de Valdivia. Se trata del inicio de año escolar en Chile, cuando los niños vuelven de vacaciones de verano y sus actividades escolares recién se han iniciado con dos o tres semanas de antelación. Los estudiantes tienen entre 15 y 17 años y todos cursan el segundo año de educación secundaria (Educación Media). 
Materiales e Instrumentos. Los sujetos completaron el programa informático "Rotacion. exe" (Castelló y Cladellas 2000). El software se compone de 64 diagramas en forma de pequeños mapas (ver figura 5). Las imágenes se presentan en el centro de la pantalla del ordenador. Ante cada una de ellas el sujeto debe situarse mentalmente en la entrada para luego decidir si debe girar hacia la derecha o izquierda según el punto en que se encuentre la salida, pulsando las teclas del ordenador identificadas con adhesivos I (izquierda) y D (derecha). Los puntos de entrada y salida se encuentran siempre formando un ángulo de $90^{\circ}$, pero la posición de las mismas varía según la figura, siendo necesario en algunas situaciones realizar un proceso de rotación mental.

\section{Figura 5}

Ejemplo de diagramas de resolución espacial
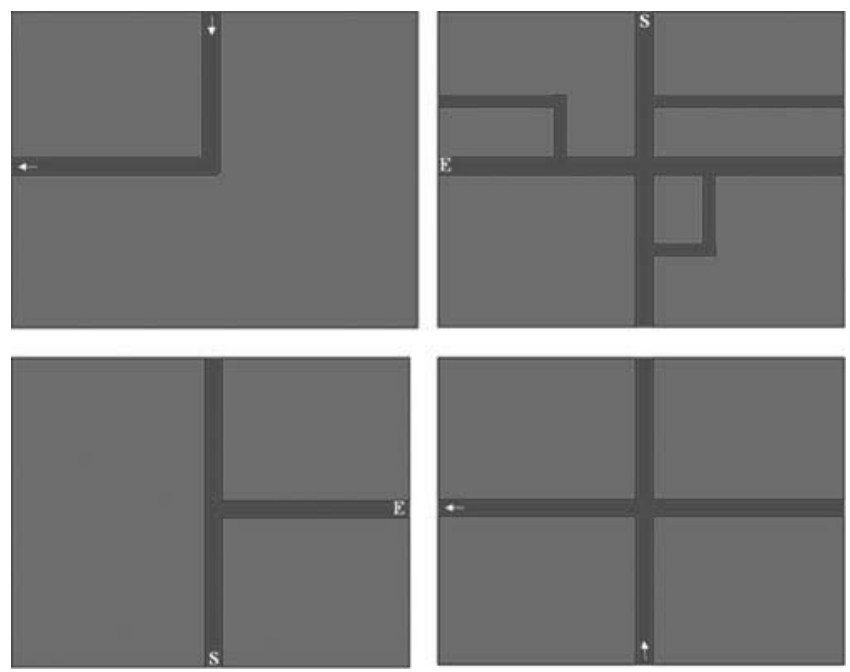

Fuente: Elaboración propia

El programa registra todos los movimientos de los sujetos y permanece activo hasta completar 180 segundos. Momento en que los sujetos harán la estimación de tiempo transcurrido.

Se emplearon ordenadores (Pentium $41.80 \mathrm{GHz}$ ) situados en el laboratorio de la RED Enlaces de los colegios, para que las condiciones experimentales fueran conocidas e iguales para todos los participantes.

En el caso de la prueba de papel se elaboraron cuadernillos con las mismas secuencias de 64 figuras que posee el software, desarrollado anteriormente por Castelló y Cladellas (2000) "rotacion.exe". Los sujetos marcarán las soluciones a los diagramas en una hoja de respuesta tachando con una cruz las opciones I (izquierda) y D (derecha). Luego de cronometrados 180 segundos de trabajo en papel, los sujetos debían anotar en la misma hoja de respuesta el tiempo estimado en minutos y segundos. 
Procedimiento. La actividad experimental consiste en ejecutar una tarea espacial de resolución de diagramas en forma de laberintos durante 180 segundos. Se eligió este tipo de tarea ya que puede considerarse neutra frente a su ejecución en papel u ordenador. Finalizada la actividad, cada sujeto anotaba el tiempo que estimaba había transcurrido en minutos y segundos.

Por un lado, contamos con un pequeño software que presenta 64 diagramas. En cada uno, los sujetos deben situarse en el punto marcado como entrada en el diagrama y elegir la dirección de salida del mismo. Acción que se ejecuta pulsando dos teclas previamente marcadas "I" de izquierda o "D" de derecha.

La ejecución de la tarea en papel implica exactamente las mismas 64 figuras ordenadas en un cuadernillo de papel. Las soluciones para cada diagrama se registrarán en una hoja de respuesta en la que los sujetos marcarán con una cruz las letras "I" o "D", Izquierda o Derecha, respectivamente.

A modo de evitar efectos indeseables y mantener el control sobre el orden de ejecución de las tareas (PC y papel) se decidió que cada uno de los 58 sujetos ejecutaría las dos tareas. Una primera mitad lo haría en la secuencia PC/papel y la segunda mitad lo haría en la ordenación papel/PC. Por supuesto se mantendrá el equilibrio de género (hombres y mujeres) y procedencia (rurales y urbanos).

Tabla 2

Conformación de Grupos Experimentales

\begin{tabular}{|l|l|}
\hline $\begin{array}{l}\text { Grupo 1 } \\
\text { Procedencia: Rural } \\
\text { Distribución por género: } \\
8 \text { hombres y 7 mujeres. } \\
\text { Orden: Papel/PC }\end{array}$ & $\begin{array}{l}\text { Grupo 2 } \\
\text { Procedencia: Rural } \\
\text { Distribución por género: } \\
7 \text { hombres y 7 mujeres. } \\
\text { Orden: PC/Papel }\end{array}$ \\
\hline $\begin{array}{l}\text { Grupo 3 } \\
\text { Procedencia: Urbano } \\
\text { Distribución por género: } \\
7 \text { hombres y 7 mujeres. } \\
\text { Orden: Papel/PC }\end{array}$ & $\begin{array}{l}\text { Grupo 4 } \\
\text { Procedencia: Urbano } \\
\text { 8 hombrión y 7 mujeres. } \\
\text { Orden: PC/Papel }\end{array}$ \\
\hline
\end{tabular}

Los grupos de sujetos harán una estimación del tiempo que transcurre mientras ejecutan una tarea sin y con asistencia tecnológica de un ordenador. Cuidando invertir equivalentemente entre grupos el orden de pasación de las pruebas (binomios papel-PC y PC-papel) y la procedencia cultural de los sujetos (ruralidad y urbanidad). Los sujetos voluntarios para este experimento no son el resultado aleatorio de la población de adolescentes chilenos, sino que pertenecen a tres colegios colaboradores de la provincia de Valdivia en la Región de Los Lagos en el sur de Chile.

Se trató de evaluar la estimación de tiempo ejecutando una tarea con presencia de tecnología informática y sin tecnología. Para ello, se eligió una tarea de rotación mental, 
que cuenta con una versión en software (Castelló y Cladellas 2000), realizándose -para esta experiencia- una versión similar en papel. Es importante señalar que la diferencia de formatos que es el eje de esta investigación no permite hablar de tareas iguales, sino similares.

\section{RESULTADOS}

A modo de introducción cabe señalar que este análisis contempla dos niveles de resultados. Un primer nivel descriptivo que da cuenta de la subestimación o sobrestimación del tiempo en términos muy generales. Un segundo nivel que aporta los resultados de las pruebas estadísticas para cada una de las diferentes hipótesis.

En ese segundo nivel estadístico el trabajo implica también dos tipos de análisis. En primer lugar un análisis de las diferencias (T de Student) comparando los dos formatos de realización de las pruebas (papel v/s ordenador) y como segunda comparación los grupos (muestras independientes) contrastando conjuntos como rurales v/s urbanos.

Resultados generales: Predominio de la mayor estimación de tiempo según tipo de tarea. Tanto en rurales como en urbanos, en hombres como en mujeres, la estimación de tiempo mayor recae sobre la tarea ejecutada en ordenador, donde un $62 \%$ de la muestra estimó como mayor el tiempo transcurrido ejecutando la tarea en ordenador, mientras que solamente el $22 \%$ de la muestra percibió como mayor el tiempo transcurrido cuando ejecutaban la tarea en papel y un $16 \%$ consideró que trabajó el mismo tiempo en papel que en ordenador.

\section{Gráfico 1}

Predominio de la estimación de tiempo por formatos

Total

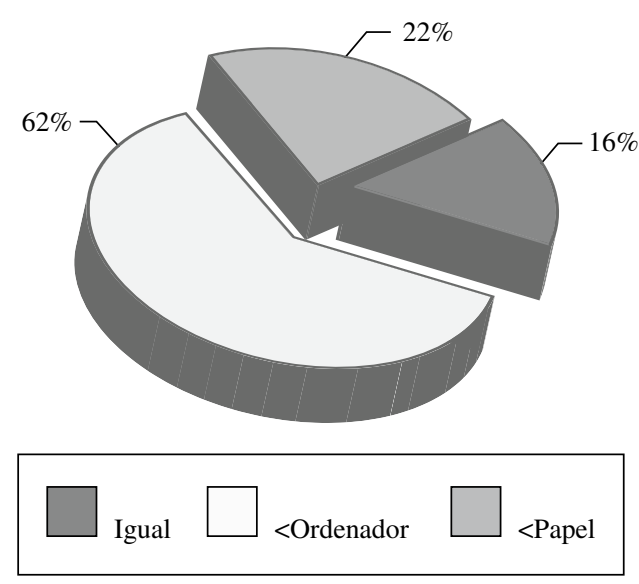


Mirando específicamente a la luz de la procedencia de los sujetos, es posible determinar que también en los urbanos un $62 \%$ de la muestra estimó como mayor el tiempo transcurrido ejecutando la tarea en ordenador, mientras que solamente el $21 \%$ de la muestra percibió como mayor el tiempo transcurrido mientras ejecutaban la tarea en papel y un $16 \%$ consideró que trabajó el mismo tiempo en papel que en ordenador.

Además, el $62 \%$ de los sujetos rurales de la muestra estimó como más largo el tiempo transcurrido ejecutando la tarea en ordenador, mientras que solamente el $24 \%$ de la muestra percibió como mayor el tiempo transcurrido cuando ejecutaban la tarea en papel y un $14 \%$ consideró que trabajó el mismo tiempo en papel que en ordenador.

\section{Gráfico 2}

Predominio de la estimación de tiempo por formatos en sujetos urbanos

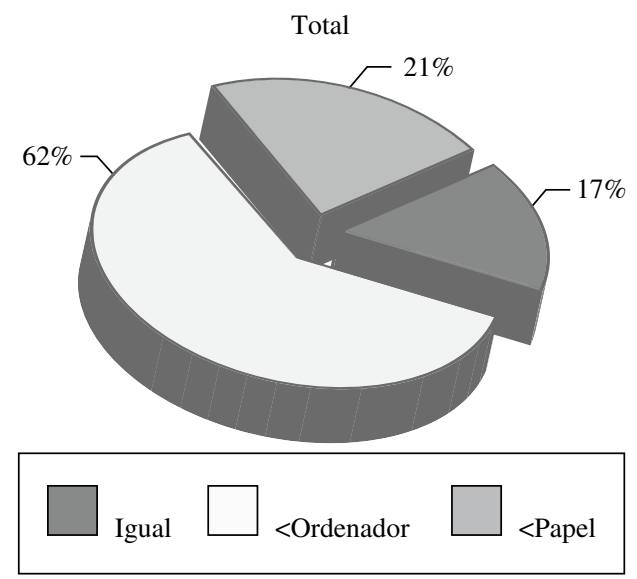

Gráfico 3

Predominio de la estimación de tiempo por formatos en sujetos rurales

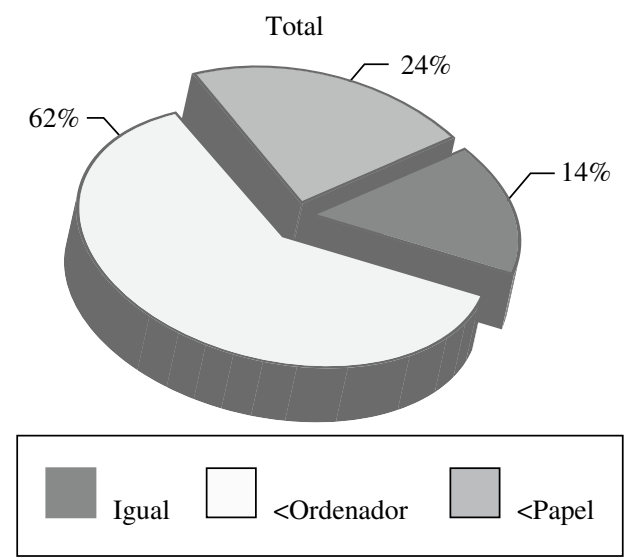


Este nivel descriptivo de los resultados sólo nos permite explicar una tendencia general a considerar como más larga la actividad ejecutada en ordenador respecto de la tarea desarrollada en papel, pero para responder a las hipótesis propuestas por esta investigación son necesarios unos análisis estadísticos más avanzados y detallados. Para ello será importante tener en cuenta que los sujetos diferían ostensiblemente en el número de diagramas resueltos cuando desarrollaban la tarea en ordenador y papel.

\section{Tabla 6}

Prueba T Diagramas resueltos en la totalidad de los sujetos

Estadísticos de Muestras relacionadas

\begin{tabular}{|l|c|c|c|c|c|c|c|}
\hline & Media & $\mathrm{N}$ & $\begin{array}{c}\text { Desviación } \\
\text { típica }\end{array}$ & $\begin{array}{c}\text { Error } \\
\text { típico }\end{array}$ & $\mathrm{T}$ & $\mathrm{gl}$ & $\begin{array}{c}\text { Significación } \\
\text { (bilateral) }\end{array}$ \\
\hline Diagramas papel & 31,33 & 58 & 13,43 & 1,76 & $-15,005$ & 57 & 0,000 \\
Diagramas ordenador & 55,79 & 58 & 10,38 & 1,36 & & & \\
\hline
\end{tabular}

Diagramas papel $=$ Número de diagramas ejecutados en prueba de papel.

Diagramas ordenador $=$ Número de diagramas ejecutados en prueba de ordenador.

Como se aprecia, la media de ejercicios ejecutada para la tarea desarrollada en papel fue 31,33 diagramas resueltos y cuando la tarea es ejecutada en ordenador se resolvieron 55,79 imágenes. Existen diferencias significativas $(\mathrm{p}<0,001)$ en los diagramas ejecutados para la tarea de rotación mental, cuando se ejecuta esta labor en el ordenador respecto al formato en el papel.

Resultados específicos de las diferencias entre formatos entre grupos. Una observación general de los datos presentados contrastando los formatos de ejecución indican que las diferencias resultan altamente significativas en todos los casos, dando cuenta así de una tendencia general. Más adelante se abordarán en detalle cada uno de estos resultados comparando las diferencias entre los formatos papel y ordenador.

En cuanto a los análisis entre grupos de muestras independientes, las diferencias no son significativas tanto para estimación de tiempo como para la cantidad de diagramas resueltos en la tarea. Por el contrario, las diferencias entre grupos es positiva a favor de la tarea realizada en ordenador en los sujetos rurales y también positiva a favor de hombres sobre mujeres en papel y ordenador cuando se evalúa la correcta resolución de los diagramas. A continuación se presentarán los resultados hipótesis a hipótesis.

Análisis por hipótesis con pruebas estadísticas para la estimación de tiempo. Para comprobar la hipótesis principal 1, si el formato de ejecución (ordenador o en papel) para una tarea espacial de resolución de diagramas incide en la estimación de tiempo que hacen los sujetos, donde la variable dependiente principal es la estimación de tiempo y las independientes corresponden al formato de aplicación del experimento (ordenador y papel), se realizó una prueba $\mathrm{T}$ de Student comparando las medias de tiempo de las muestras relacionadas. 
Tabla 3

Prueba T Diferencias Generales en la Estimación de Tiempo (formato)

Estadísticos de muestras relacionadas

\begin{tabular}{|l|l|c|c|c|c|c|c|}
\hline & Media & $\mathrm{N}$ & $\begin{array}{c}\text { Desviación } \\
\text { Típica }\end{array}$ & $\begin{array}{c}\text { Error } \\
\text { típico }\end{array}$ & $\mathrm{T}$ & Gl & $\begin{array}{c}\text { Significación } \\
\text { (Bilateral) }\end{array}$ \\
\hline Tiempo papel & 149,826 & 58 & 83,63 & 10,98 & $-4,047$ & 57 & 0,000 \\
Tiempo ordenador & 200,2241 & 58 & 110,42 & 14,50 & & & \\
\hline
\end{tabular}

Como se aprecia, la media de estimación de tiempo para la tarea ejecutada en papel es de 149,8 segundos y cuando la tarea es ejecutada en ordenador el tiempo estimado por los sujetos es de 200,2 segundos. Siendo el tiempo real de trabajo 180 segundos. Así existen diferencias significativas $(\mathrm{p}<0,001)$ en la estimación del tiempo cuando ejecutan la tarea de rotación espacial en PC y en papel.

Los resultados confirman que existe una relación entre la variable independiente formato y la dependiente estimación de tiempo. Esta situación se repite para cada uno de los subgrupos: hombres, mujeres, urbanos y rurales. En todos los casos, las diferencias siempre son significativas.

Análisis entre grupos comparando procedencia y estimación de tiempo. Para evaluar la hipótesis secundaria respecto de si la ejecución en ordenador o la ejecución en papel de una tarea espacial de resolución de laberintos incide de forma distinta en la estimación de tiempo que hacen los sujetos que provienen de contextos culturales diferentes, es posible hacer un análisis estadístico comparando sujetos rurales y urbanos, y el formato de ejecución de la prueba sea el mismo.

El formato de ejecución (ordenador o en papel) para una tarea espacial de resolución de diagramas incide de forma distinta en la estimación de tiempo que hacen los sujetos que provienen de contextos culturales diferentes (rurales y urbanos).

Para este análisis se efectuó una prueba estadística de muestras independientes que compara la estimación de tiempo entre procedencias distintas (rurales y urbanos) ante el mismo formato de ejecución del experimento.

\section{Tabla 4}

Medias de Estimación del tiempo y procedencias

Estadísticos de muestras independientes

\begin{tabular}{|l|c|c|c|c|c|}
\hline & Grupo & N & Media & Desviación Típica & P \\
\hline \multirow{2}{*}{ Papel } & Rurales & 29 & 128,66 & 76,83 & \\
& Urbanos & 29 & 171,06 & 86,06 & 0,053 \\
\hline \multirow{3}{*}{ Ordenador } & Rurales & 29 & 194,44 & 104,09 & \\
& Urbanos & 29 & 206,00 & 117,98 & 0,694 \\
\hline
\end{tabular}


Las medias de estimación de tiempo específicas de cada subgrupo de procedencias están contenidas en la tabla 4.

Si se analizan las diferencias de estimación de tiempo entre grupos (rurales y urbanos) ante un mismo tipo de tarea, la prueba de muestras independientes proporciona diferencias no significativas, tanto para la tarea realizada en papel $\mathrm{p}=0,053$ como para la tarea realizada en ordenador $\mathrm{p}=0,694$. Sin embargo, cuando la tarea se desarrolla en papel la diferencia casi es significativa y por tanto se puede pensar que si el experimento contase con una muestra mayor se incrementaría el diferencial en el intergrupo papel.

\section{Gráfico 6}

Representación gráfica de estimación del Tiempo y procedencia

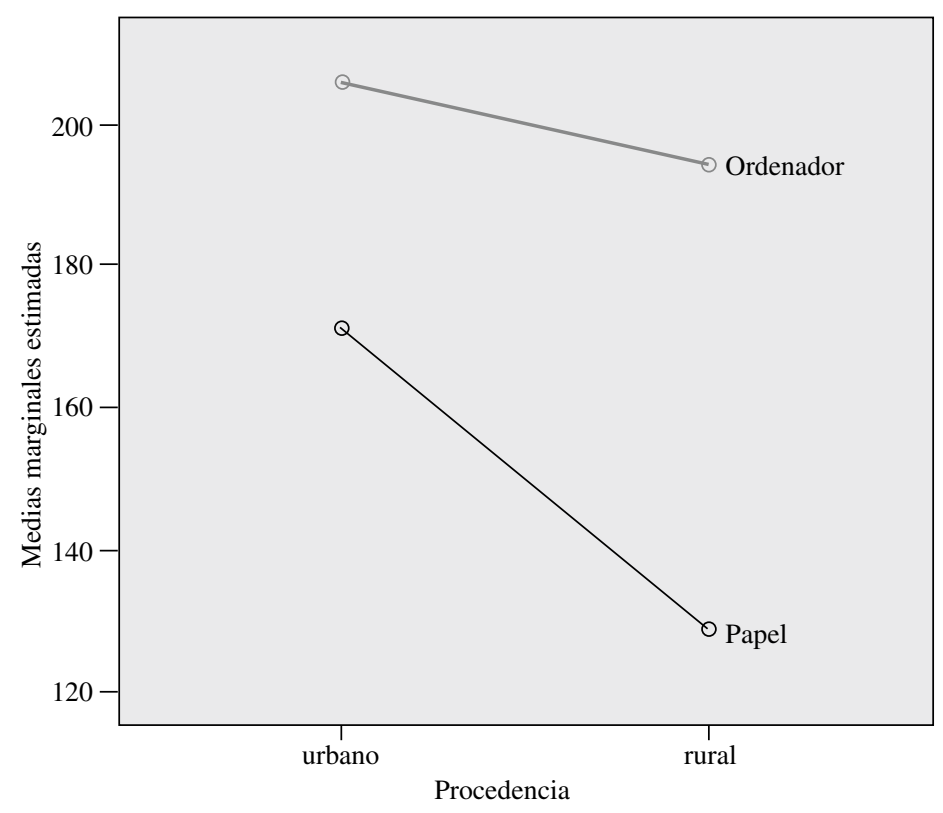

El gráfico 6 representa las medias de estimación de tiempo en segundos, desde el que se puede comprobar cómo los tiempos estimados en ordenador son mayores a los estimados en papel. Por otra parte, la mayores diferencias entre pruebas se dan entre los sujetos rurales.

\section{DISCUSION}

A continuación se tratarán los principales hallazgos respecto a cada una de las temáticas abordadas, llegando en algunos casos a conclusiones concretas y, en otros, a cuestionar determinados aspectos que bien podrían ser resueltos en futuras investigaciones. 
En concreto en este apartado se comentará a qué conclusiones se ha llegado para cada una de las hipótesis referentes a la estimación del tiempo; si bien también se tratarán aspectos no directamente relacionados con el objetivo principal del trabajo, pero que pueden ayudar a esclarecer algunos de los apartados no resueltos en esta investigación.

Los datos obtenidos a través de este estudio demuestran que la estimación del tiempo es siempre menor cuando la tarea espacial es desarrollada en formato papel frente a la misma desarrollada en formato ordenador. Por tanto, se puede concluir que el formato tecnológico incide altamente en la percepción del tiempo, en concreto en lo que se refiere a la estimación del tiempo empleado en el desarrollo de la tarea.

Teniendo la tarea, tanto en ordenador como en papel, una duración de 180 segundos, la estimación del tiempo efectuada por los sujetos experimentales puede resumirse en que sólo un $15 \%$ de los sujetos son capaces de estimar como iguales los tiempos empleados en la tarea de ordenador y papel. Un 60\% estima como mayor el tiempo transcurrido en ordenador y un $25 \%$ experimenta un tiempo más largo cuando ejecutan la tarea en papel.

Aunque estos resultados pueden parecer contradictorios con supuestos aspectos motivacionales que podrían subestimar el paso del tiempo, son totalmente congruentes con la explicación de que la sobreestimación del tiempo puede venir provocada por la mayor cantidad de ejercicios realizados en ordenador. De alguna manera, cada diagrama realizado va segmentando el tiempo vivido por los sujetos. Tal y como señalan los estudios de Pointer (1989), el mayor número de cambios experimentados en 180 segundos cuando realizan la prueba en ordenador produciría el efecto de prolongar la estimación del tiempo.

A la luz del modelo de Morales (2005) se está ante una duración prolongada del tiempo, donde la dimensión influyente sería la percepción de la carga de información. Los sujetos estudiados configuran la estimación del tiempo contemplando los cambios de diagramas resueltos en ordenador, que por lo general casi duplica (57 versus 31 ) a las resoluciones en papel. Con ello, el número de diagramas produce la segmentación del tiempo y una sobreestimación frente a una tarea en la que los cambios experimentados son aproximadamente la mitad (papel).

La hipótesis principal que proponía el presente trabajo experimental, donde el formato de ejecución de la tarea en ordenador incide en una percepción de duración prolongada del tiempo estimado, es confirmada por la experimentación.

En síntesis, lo relevante a tener en cuenta es que cuando un sujeto se muda desde la manualidad a la realización de una tarea similar en ordenador, no necesariamente se sentirá más aliviado. Si cuando desarrolla la tarea en ordenador experimenta un número ostensiblemente mayor de subintervalos, el sujeto es consciente de que realiza más ejercicios y por tanto tiende a sobreestimar el tiempo, siendo esta situación coherente con el modelo change/segmentation de Pointer (1989).

Con respecto a la relación de la variable formato con la variable procedencia cultural, las diferencias no son significativas para la estimación del tiempo y con ello se rechaza la hipótesis secundaria que se planteaba, donde los sujetos rurales menos impactados tecnológicamente estimarían como menor el tiempo transcurrido. Pero la $\mathrm{p}=0,053$ encontrada entre la población rural y urbana cuando la tarea se desarrolla en formato papel, hace pensar que el factor cultural tiene un peso considerable, aunque no determinante en la estimación del tiempo y que, con una muestra más grande, las diferencias seguramente serían significativas. 
Ante el fenómeno expuesto, el esquema de Morales (2005) define una situación de compresión temporal, donde los sujetos rurales estiman en aproximadamente 2 minutos una tarea que en realidad dura 3 minutos, situación que no se produce en los sujetos urbanos. El modelo de Morales debería explicar el hecho atendiendo a un mayor dominio de la tarea o a una mayor alerta. La primera situación es descartable, ya que la tarea es ejecutada con precisión por ambos grupos (21 diagramas como media, tanto para rurales como para urbanos). Entonces cabría proponer que la alerta sería mayor en los rurales, pero el modelo no permite responder por qué sucede así; además, la alerta de las situaciones experimentales son equivalentes: las instrucciones y materiales (cuadernillos) son los mismos.

La pregunta que nos planteamos es: ¿los sujetos rurales están realmente más alertas? ¿Podría ser que los sujetos urbanos se encontrasen menos motivados al realizar la tarea en papel?

También se puede entender como normal que la mayor estimación del tiempo por parte de los urbanos podría ser consecuencia de que realizar una tarea en un formato obsoleto y poco usual, en una sociedad totalmente tecnológica, la convierte en una tarea aburrida y poco motivadora.

Siguiendo con lo anterior es algo que no debe extrañar, si tenemos en cuenta que en las sociedades urbanas el empleo del ordenador para la práctica educativa y de ocio se ha convertido en una herramienta imprescindible; mientras que en las sociedades rurales el uso del ordenador todavía sigue siendo algo excepcional, y en los pocos casos en que se emplea de forma habitual no deja de ser algo con un pasado muy reciente. Todo ello aún cobra mayor importancia, si tenemos en cuenta que estamos hablando de adolescentes de un país como Chile (en que las diferencias entre población rural y urbana aún siguen siendo muy evidentes), donde el impacto de la tecnología en la ruralidad puede haber llegado a las escuelas, pero permanece ausente en la cultura del ocio, en la que mayormente persisten las prácticas al aire libre.

En conclusión, en relación a las diferencias culturales, la situación experimental invita a contemplar para futuras investigaciones la medición del factor motivacional como factor más explicativo que la alta o baja alerta propuesta por Morales (2005) de Tiempo Psicológico en Estudiantes Usando Tecnología.

\section{REFERENCIAS}

Beas, J. (2003). ¿Qué debemos enseñar y qué debemos aprender en la escuela del siglo XXI? Revista Pensamiento Educativo 32 (2): 49-70.

Castells, M. (1997). La era de la información: economía, sociedad y cultura: La sociedad red vol. 1, Madrid: Alianza Editorial.

Castells, M. (2001). La Galaxia Internet. Barcelona: Editorial Arete.

Castelló, A. y R. Cladellas (2000). Software informático "Rotación.exe”. Barcelona: Universidad Autónoma de Barcelona. (CD Room. No Publicado).

Choufhury, N. y K. Gorman (1999). The relationship between reaction time and psychometric intelligences in a rural Guatemalan adolescent population. International Journal of psychology, 34 4: 209-217.

Enlaces. (2005). Cultura Digital en el sistema escolar chileno. Santiago de Chile: EnlacesMinisterio de Educación. 
Enlaces. (2005). Educación en la sociedad de la información. Santiago de Chile: Collect, EnlacesMinisterio de Educación.

Fraisse, P. (1973). Percepción y Estimación del Tiempo. En Piaget y Fraisse y coords. Tratado de Psicología Experimental, Cap. II, vol 6. Argentina: Buenos Aires, Paidós Editorial.

Gurevitch, A.Y. (1979). El tiempo como problema de historia cultura. En Las culturas y el tiempo (pp. 260-281). París: Sígueme, UNESCO.

Hall, E. (1989). El lenguaje Silencioso. España: Madrid, Alianza Editorial.

McLuhan, M. (1990) La Aldea Global: Transformaciones en la vida y en los medios de comunicación mundiales en el siglo XXI. España. Barcelona, Editorial Gedisa.

Morales, C. (2005). Tiempo psicológico en los estudiantes y carga de información. Revista Ingeniería, abril-junio, Vol. VIII, $\mathrm{N}^{\circ} 27$. México. Facultad de Ingeniería Mecánica y Eléctrica de la Universidad Autónoma de Nuevo León.

Orsini, F. (1971). Contribución al estudio genético de la estimación del tiempo en función de la variación de las situaciones. En La Epistemología del Tiempo (Piaget, 1971. cord). Capítulo $V$. Argentina: Buenos Aires, Editorial El Ateneo. Colección Biblioteca Nuevas Orientaciones de la Educación.

Orstein, R.E. (1969). On the experience of time. USA: Penguin, Harmondsworth.

Piaget, J. (1973). El desarrollo de la noción de tiempo en el niño. México: Fondo de Cultura Económica.

Piaget, J., F. Orsini M. Meylan-Backs(1971). La Epistemología del Tiempo. Argentina: Editorial El Ateneo.

Piaget, J. y M. Meylan-Backs (1971). Comparación de operaciones temporales en relación con la velocidad y frecuencia. En J. Piaget y cols, La Epistemología del Tiempo. Capítulo II. Argentina: Editorial El Ateneo. Colección Biblioteca Nueva Orientaciones de la Educación. Buenos Aires.

Poynter, W.D. (1983). Judging the Duratión of time Intervals: A process of remembering segments of experience. In Levin, I and Zakay, D. (1989) Time and Human Cognition. Netherland: Advanced In Psychology.

Schwartz, E. (2000). Darwinismo Digital. Estrategias ganadoras para sobrevivir en la asesina economía de la web. Argentina: Ediciones Granica S.A.

Toledo, E. y S. Comba (2002). El Hombre y la máquina: nuevas prácticas profesionales. Revista TELOS. Cuadernos de Comunicación, Tecnología y Sociedad 52: 22-26. 